\title{
Medicina tradicional e populações da Amazônia: realidades das políticas de saúde
}

Traditional medicine and populations of the Amazon: realities of health policies

Medicina tradicional y poblaciones de la Amazonía: realidades de las políticas de salud

Nádile Juliane Costa de Castro ${ }^{1 *}$, Antônio Luís Parlandin dos Santos ${ }^{1}$, Shirley Aviz de Miranda², Thais do Socorro da Luz Silva ${ }^{1}$, Karytta Sousa Naka ${ }^{3}$, Deisiane da Silva Mesquita ${ }^{4}$, Max José de Aviz Miranda ${ }^{5}$, Juliana Pereira Pinto Cordeiro ${ }^{6}$, Juliana Costa de Castro ${ }^{7}$, Dayanne de Nazaré dos Santos ${ }^{8}$.

\section{RESUMO}

Objetivo: Revisar na literatura a respeito das políticas de saúde direcionadas a medicina tradicional em um município paraense. Métodos: Estudo de abordagem exploratória e descritiva, com caráter documental. Como aporte foi realizada a busca de documentos oficiais, artigos científicos e sites oficiais com relação direta com o município estudado. Resultado: Encontrou-se 06 publicações entre os anos de 2006 a 2018. Nestas foram identificadas ações direcionadas a Política Nacional das Práticas Integrativas e complementares em saúde nos serviços e instituições locais. Há ações paralelas relacionadas a ampliação das ações com Universidades Públicas. Conclusão: A política está sendo implantada como projeto piloto no município em parceria a instituições de ensino, contudo sua manutenção é deficiente em função do não comprometimento dos profissionais, déficits de acesso a serviços de saúde em algumas localidades e de conhecimento dos técnicos em saúde. A realidade demostra as dificuldades para manutenção e ampliação das políticas públicas de saúde em virtude das peculiaridades da Amazônia apesar da legislação fundamentar sua execução.

Palavras-chave: Medicina Tradicional, Serviços de Saúde, Políticas Públicas, Legislação em Saúde.

\begin{abstract}
Objective: To review in the literature about the health policies directed to traditional medicine in a municipality of Pará. Methods: Exploratory and descriptive study, with documentary character. As a contribution was made the search for official documents, scientific articles and official sites with direct relation with the municipality studied. Results: There were 06 publications between the years 2006 and 2018. These actions identified the National Policy on Integrative and Complementary Practices in Health in local services and institutions. There are parallel actions related to the expansion of actions with Public Universities. Conclusion: The policy is being implemented as a pilot project in the municipality in partnership with educational institutions, but its maintenance is deficient due to the lack of commitment of professionals, deficits in access to health services in some localities and knowledge of health technicians. The reality demonstrates the difficulties to maintain and expand public health policies due to the peculiarities of the Amazon, despite the legislation underlying its execution.
\end{abstract}

Keywords: Traditional Medicine, Health Services, Public Policies, Health Legislation.

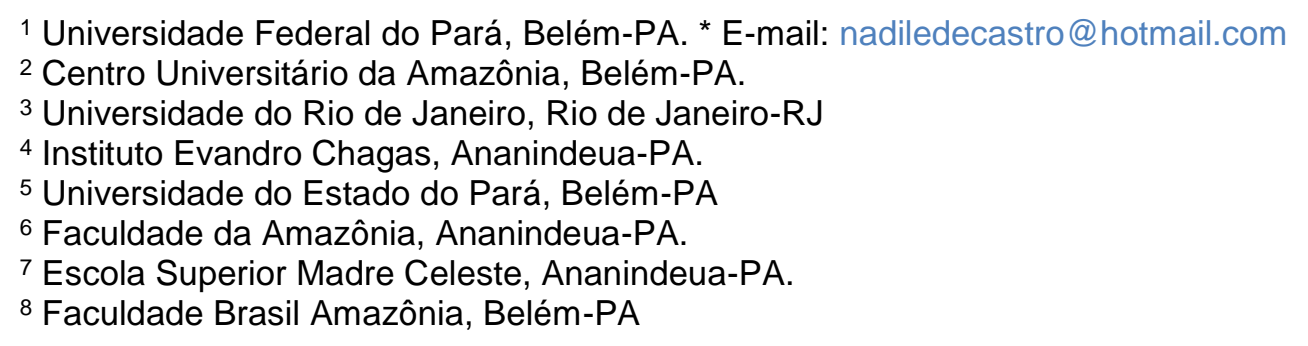




\section{RESUMEN}

Objetivo: Revisar en la literatura acerca de las políticas de salud dirigidas a la medicina tradicional en un municipio paraense. Métodos: Estudio de abordaje exploratorio y descriptivo, con carácter documental. Como aporte se realizó la búsqueda de documentos oficiales, artículos científicos y sitios oficiales con relación directa con el municipio estudiado. Resultado: Se han encontrado 06 publicaciones entre los años 2006 a 2018. En estas se identificaron acciones dirigidas a la Política Nacional de las Prácticas Integrativas y complementarias en salud en los servicios e instituciones locales. Hay acciones paralelas relacionadas con la ampliación de las acciones con Universidades Públicas. Conclusión: Una política está implantada como un proyecto piloto en un plan de acción y en un futuro, sin duda alguna, se trata de una función de compromiso y un compromiso profesional, un conjunto de servicios y servicios en su conjunto. Una realidad demostrable como es difícil para la administración y la ampliación de las políticas públicas de las virtudes de las peculiaridades de la Amazonía y la legislación de su país.

Palabras clave: Medicina Tradicional, Servicios de Salud, Políticas Públicas, Legislación en Salud.

\section{INTRODUÇÃO}

A Declaração da Alma-Ata (OMS, 1978) foi um teve um papel importante nos adventos da saúde, haja vista que compõem-se de 10 itens que enfatizam a atenção primária em saúde e se constituiu um instrumento crucial para o reconhecimento dos cuidados de saúde primários, o que fortaleceu o processo e implantação de suas diretrizes nos diversos cenários de saúde em todo o mundo. Por certo, a legislação brasileira deu subsídios para sua execução incluindo os debates da $8^{\text {a }}$ Conferência Nacional de Saúde (CNS). Já a Lei Orgânica do Sistema Único de Saúde (SUS) de 1990 veio concretizar estas iniciativas por meio de seus objetivos e diretrizes, sendo constantemente implementada para alcançar resultados satisfatórios.

Entre as Políticas de saúde criadas nestes quase 30 anos do SUS houve em 2006 a criação da Política Nacional de Práticas Integrativas e complementares (PNPIC). É uma política em processo contínuo de inclusão de práticas alternativas e complementares em saúde, datada de 2018 sua última atualização, sendo, portanto, necessária para fortalecer os conhecimentos tradicionais e para o desenvolvimento de tratamentos alternativos no SUS (BARROS NF, et al.,2018). A inserção de políticas envoltas a medicina tradicional é um movimento observado em todo Brasil e tem apresentado versos resultados sejam eles positivos ou não, principalmente dentro da atenção primária em saúde do sistema de saúde público brasileiro. Contudo, apresenta-se como um desafio frente a gestão e equipes multiprofissionais quando da sua implementação (PLÁCIDO AL et al., 2019).

Questões relativas ao déficit de conhecimento dos profissionais de saúde perante aos tratamentos e a descredibilidade destas alternativas tem sido as mais citadas entre os pesquisadores da área de trabalho em saúde (GONTIJO MBA e NUNES MF, 2017; SILVA ASP e FEITOSA ST, 2018). Certamente há várias justificativas para estes achados, o que deveras ser sempre observado na implementação desta política dentro dos serviços de atenção à saúde, abrindo espaço para discussão entre os envolvidos no processo a fim de identificar as melhores alternativas para resultados satisfatórios. Por certo, é necessário construir essas ações pela compreensão de cada realidade por meio dos cenários, suas dinâmicas e peculiaridades.

É uma política de saúde relevante dentro do cenário da Amazônia, haja vista que é uma região que possui um extensa biodiversidade, povos, populações e comunidades que apresentam particularidades quanto das crendices, costumes e valores que são repassados entre as gerações (PEREIRA MJS e COELHO-FERREIRA $M, 2017)$. De todo modo é indiscutível a importância desta política inclusive como meio para o protagonismo de povos e populações frente seus conhecimentos e possibilitando que no seu itinerário terapêutico seja considerado suas escolhas pelo seu modo de vida.

Por certo, o fortalecimento de práticas alternativas no SUS possibilita que por meio destes saberes a interrelação entre profissionais e comunidades seja mais estreita. Certamente que o ponto inicial é diminuir os vários problemas apontados pelas ações já observadas em todo o país, inclusive da medicalização imposta pelo modelo biomédico (FEITOSA ST, 2018). Contudo, percebe-se pelos seus delineamentos que há o fortalecimento da atenção básica, estimula o controle e participação social, além de estimular "alternativas 
inovadoras e socialmente contributivas ao desenvolvimento sustentável de comunidades" como prevista na legislação da PNPIC (BRASIL, 2006; BRASIL, 2015).

Nestes termos, há de considerar-se investigar cenários da Amazônia que apresentam essas configurações quanto a biodiversidade, povos e populações. Diante disso, buscou-se fazer a leitura de Oriximiná, município situado na região oeste da Amazônia paraense, caracterizado por uma população de aproximadamente 63 mil habitantes entre estes há indígenas e remanescentes quilombolas (IBGE, 2017).O município de Oriximiná possui 21 aldeias, aproximadamente 2.700 indígenas que são atendidos pelo Polo Indígena de Oriximiná pertencente ao Distrito Guamá Tocantins por meio da Secretaria Especial de Saúde Indígena do Ministério da Saúde (BRASIL, 2018).

Logo, como propósito de discutir frente a realidade local, o objetivo do trabalho é revisar a literatura a respeito das políticas de saúde direcionadas a medicina tradicional neste município paraense e discuti-lo frente as políticas públicas de saúde.

\section{MÉTODOS}

Trata-se de um estudo de abordagem exploratória e descritiva, com caráter documental. Como aporte foi realizada a busca de documentos oficiais, artigos científicos com relação direta com o município estudado. Foram identificadas e analisadas 06 publicações entre os anos de 2006 a 2018, considerando-se os disponíveis e publicados em revistas científicas, dados com informações por meio de sites oficiais e com relação direta com o município estudado. Quando da busca de artigos foi realizada na base de dados Scielo, LILACS e Google Scholar. A busca dos artigos foi feita por meio de associação do descritor: Políticas Nacional de práticas integrativas e complementares, Fitoterapia e Medicina Tradicional. Destaca-se que inicialmente todos os artigos foram recuperados, não sendo eliminada nenhuma publicação a priori.

Ao final da seleção de bibliografias, constituiu-se como objeto de análise constituído de documentos oficiais, artigos e informações de sites oficiais do governo municipal, que foram fundamentais para a construção das reflexões. Destaca-se que os artigos selecionados abordavam pesquisas em diferentes áreas. Isto promove o direcionamento para um diálogo que seja interdisciplinar abrangendo diversos campos, mas prioritariamente neste estudo ao campo da saúde coletiva.

Procurou-se fundamentar, ainda, por meio da legislação de modo que quando analisadas os achados ampliassem a discussão e justificassem a importância desta política de saúde para a acesso dos serviços e a integralidade da assistência. Nestes termos, foram criados os seguintes eixos temáticos: Contextos da PNPIC entre povos e populações de Oriximiná e Conhecimento e questões jurídicas.

\section{RESULTADOS E DISCUSSÃO}

\section{Contextos da incorporação da PNPIC entre povos e populações de Oriximiná}

Oriximiná apresenta-se entre os 88 municípios do Estado do Pará que utiliza práticas integrativas no SUS com destaque para as plantas medicinais e fitoterapia segundo o Ministério da Saúde (BRASIL, 2018a). A iniciativa de implantação compete ao gestor municipal que deve reconhecer a PNPIC e principalmente as seguintes diretrizes: Elaboração da Relação Nacional de Plantas Medicinais e da Relação Nacional de Fitoterápicos; Provimento do acesso a plantas medicinais e fitoterápicos aos usuários do SUS; Formação e educação permanente dos profissionais de Saúde em plantas medicinais e fitoterapia; Acompanhamento e avaliação da inserção e implementação das plantas medicinais e fitoterapia no SUS (BRASIL, 2015).

O olhar do gestor deve pautar na participação social, e sobretudo buscar parcerias para a execução destas políticas, de modo que haja cooperação entre entidades de diversos fins, assim como de uma gestão matricial. De fato, isso vem sendo executado no município, haja vista que há ações que envolvem ensino-serviço e 
comunidade e que tem favorecido a implantação da PNPIC. No mais, essa configuração promove trocas de experiências entre os profissionais de saúde, o gestor, comunidade e pesquisadores, assim como possibilita dentro do ensino uma formação comprometida e capaz de atuar de modo a perceber necessidades das populações da Amazônia, sobretudo quando inserida em uma educação comprometida (DE CASTRO NJC, et al., 2017)

Nesta direção há em Oriximiná a execução de um projeto intitulado "Fitorixi" que está vinculado a Universidade do Oeste do Pará (UFOPA) e ao projeto "Plantas Medicinais no SUS" e tem como objetivo implantar ações por meio de pesquisa-ação envolvendo conhecimento tradicional a partir de remédios caseiros com origem no bioma amazônico. Para efetivação de seus objetivos foi realizada parceria com a Secretaria Municipal de Saúde através das Unidades básicas de Saúde e busca-se ampliar as possibilidades de tratamento alternativos e novidades que tragam benefícios aos usuários do SUS, mas sobretudo, ampliar discussões sobre a conservação da biodiversidade por meio da saúde coletiva e empoderar os conhecimentos tradicionais locais (LEDA PHO, et al., 2017; LÉDA PHO, et al., 2018).

A metodologia deste trabalho é baseada na etnobotânica com participação inicial de discentes de biologia e agentes comunitários de saúde de Oriximiná e demostrou as relações intergeracionais atreladas ao conhecimento de idosos e falta de credibilidade e confiança dos ACS em indicar tratamentos nesse patamar (LEDA PHO, et al., 2017). É certamente um projeto inovador e que tem promovido ações essências para a saúde coletiva por meio de discussões e ações agroecológicas. Outro cenário importante a considerar é a consolidação em uma Unidade Básica de Saúde que tem como referência a implantação de horta comunitária via este projeto.

Em relação as populações indígenas, foi identificado um projeto pioneiro de horta comunitária em algumas aldeias. Esta ação é uma resposta as observações dos profissionais de saúde sobre os hábitos alimentares destes povos, haja vista que os alimentos industrializados se encontram entre as atuais escolhas dentro da dieta destas comunidades. Este projeto piloto vem como estratégia de diminuir o consumo destes produtos, diminuir os agravos e possibilitar por meio da produção de alimentos a inclusão social, produtiva e a promoção da saúde (BRASIL, 2018b). É uma proposta inicial, mas que prevê a ampliação dos gêneros utilizados, mas que pode ser aporte para futuras hortas medicinais, de modo a resgatar a medicina tradicional indígena.

Já relativo as populações remanescentes quilombolas há várias situações envoltas ao projeto de mineração da bauxita. Refere-se isto porque há um projeto de resgate da cultura e formação e capital social entre esse grupo capitalista e os remanescentes quilombolas, o projeto é intitulado "territórios sustentáveis" sendo seu patrocinador a Mineração Rio do Norte.

No entanto, não há atividades até o momento ligadas e executadas nos serviços de saúde e pela medicina tradicional por este projeto. É um cenário diferente do observado na atenção à saúde dos povos indígenas, organizado pela SESAI, e das populações da zona urbana. Isso demostra que o planejamento da gestão municipal é focado ainda na população que tem acesso aos serviços de atenção básica urbana, mas que também é resultado da ausência de equipamentos de saúde na zona rural onde poderia ser implantando a PNPIC.

\section{Conhecimento e questões jurídicas}

Como exposto, a legislação em saúde tem dado subsídios para que as políticas de atenção à saúde sejam executadas, e diante das práticas populares em atenção à saúde, a legislação tem avançado para garantia de direitos sociais estabelecido no art. 6으 da Carta Magna. Os direitos sociais seriam aqueles a qual é direcionado a inclusão de pessoas a uma vida social, visando o "bem-estar" da pessoa, e com uma preocupação com a "camada mais carente da população", que não pode obter certos serviços ou benefícios de modo independente (MOTTA FILHO SC, 2003). Desta forma, o art. 6o da Constituição Federal se preocupou em proteger os mais fracos, estabelecendo através do texto constitucional, uma forma para garantia de vida digna, através do direito à saúde com previsão no art. 196 da Constituição. 
Ao compreender estes apontamentos e diante do cenário em estudo que inclui povos e populações que historicamente possuem questões sociais envoltas a sua realidade, é necessário oportunizar por meio dessas leis o direito à saúde, incluindo o direito de um itinerário terapêutico que considere seus conhecimentos populares.

Neste ensejo, a constituição ao se preocupar com a saúde da população, estipulou a garantia à saúde mediante políticas públicas e econômicas, previstas no art. 196 da Constituição Federal, e para previsão positiva dessas ações, estabeleceu no art. 3o da Lei ํo 8.080/90, a possibilidade de ações para garantia de bem-estar, físico, mental e social que são fatores determinantes e condicionantes da saúde. Essas ações são expressas no texto da carta maior, no art. 198, inciso II, que estabelece o atendimento integral, com prioridade para as atividades preventivas.

Ainda, diante da manifestação da Organização Mundial da Saúde sobre a medicina tradicional, complementar e alternativa nos sistemas de saúde, esta passou a ser incorporada no Brasil via PNPIC, através da Portaria no 971, de 03 de maio de 2006. Certamente foi um avanço quanto as possibilidades de escolhas, pois, atestou a inclusão da prática de Medicina Tradicional Chinesa-Acupuntura, Homeopatia, Plantas Medicinais e Fitoterapia e o Termalismo Social/Crenoterapia entre outras. Mas sobretudo suas diretrizes direcionam como o gestor, profissionais e comunidade podem articular-se para implantá-las, o que tem acontecido em Oriximiná.

No caso da Política Nacional de Plantas Medicinais e Fitoterápicos (PNPMF), esta foi regulamentada mediante o Decreto no 5.813, de 22 de junho de 2006. A regulamentação da PNPMF, visou além de inserir as plantas medicinais, fitoterápicos e serviços de Phytotropin no SUS a inclusão de práticas populares e tradicionais. Foi uma conquista de grande valor para o Brasil, mas principalmente para a região Amazônica, haja vista que é um país que detém a maior parcela da biodiversidade mundial, em torno de $15 \%$ a $20 \%$ (BRASIL, 2006). Logo, como há interesse nacional em promover e reconhecer as práticas populares e tradicionais, a participação de instituições de ensino e pesquisa são fundamentais. No entanto, apesar disso, questões burocráticas têm impedido que algumas instituições façam estudos com as populações de Oriximiná (OLIVEIRA DR, et al., 2013).

Certamente que o impulso para que a medicina popular fosse reconhecida ocorreu devido o Brasil tornarse signatário da Convenção sobre a Diversidade Biológica (CDB) (BRASIL, 1988), que foi promulgado sob Decreto no 2.519 de 1998. O CDB é um acordo estabelecido aos países que fazem parte da Organização das Nações Unidas. Este acordo tem como objetivo a conservação da diversidade biológica e a utilização sustentável de seus componentes. E neste mesmo viés que a CDB reforça a importância de uso de conhecimento tradicionais.

Não obstante, apesar da convenção manifestar como um dos objetivos o uso sustentável de componentes e fomentar o conhecimento tradicional, o uso de medicamentos fitoterápicos devem estar adequados ao Regulamento Técnico para Medicamentos Fitoterápico, haja vista que para ser considerado medicamento fitoterápico, este não pode ser de qualquer origem, conforme a resolução o 48 de 16 de março de 2004 (BRASIL, 2004). E com os pressupostos legais do uso da medicina popular, e as devidas a concretização da possibilidade de acesso a medicamentos, torna-se viável a implementação da promoção a saúde.

Diante do exposto, não se pode desconsiderar os direitos destes povos e populações quanto a preservação de seus saberes, pois, a apropriação de saberes populares tem sido evidenciada hodiernamente. Oriximiná possuem riquezas não somente em suas plantas e minérios, há uma riqueza de saberes e fazeres entre seus munícipes que devem ser protegidos a fim de proteger inclusive como patrimônios imateriais. Certamente que deste a Constituição Federal a proteção nestes termos vem sendo citada e as políticas e programas foram dando suporte para proteção do indivíduo, assim como do direito à saúde. 


\section{CONSIDERAÇÕES FINAIS}

O estudo contribuiu para ampliar o conhecimento acerca da medicina tradicional em populações da Amazônia paraense versus a política nacional de práticas integrativas e complementares. A análise dos dados revelou a necessidade de implementar esta política no município, haja vista que é um processo que está presente, porém não há replicação do projeto piloto implantado em outras unidades de saúde. No mais, o município representa a diversidade étnica, cultural e da biodiversidade da Amazônia paraense e tem sido alvo de estudos de etnobotânica por instituições de ensino e pesquisa.

\section{REFERÊNCIAS}

1. BARRETO JPL. Bahserikowi-Centro de Medicina Indígena da Amazônia: concepções e práticas de saúde. Amazônica-Revista de Antropologia, 2018; 9(2): 594-612.

2. BARROS NF, et al. Trabalho interprofissional e as Práticas Integrativas e Complementares no contexto da Atenção Primária à Saúde: potenciais e desafios. Saúde debate, Rio de Janeiro. 2018; 42(1):163-173.

3. BRASIL. Decreto № 2.519, de 16 DE março de 1998. Promulga a Convenção sobre a Diversidade Biológica. Rio de Janeiro, em 05 de junho de 1992.

4. BRASIL. Ministério da Saúde. Iniciativa do DSEI GUATOC implementa horta comunitária. 2018. Disponível em: http://portalms.saude.gov.br/noticias/agencia-saude/42740-iniciativa-do-dsei-guatoc-implementa-horta-comunitaria-em-areaindigena. Acesso em: 20 nov. 2018.

5. BRASIL. Ministério da Saúde. Municípios que utilizam práticas integrativas no tratamento de pacientes do SUS. 2018. Disponível em: http://portalms.saude.gov.br/noticias/agencia-saude/42808-no-para-88-municipios-utilizam-praticasintegrativas-no-tratamento-de-pacientes-do-sus.

6. BRASIL. Ministério da Saúde. Secretaria de Ciência, Tecnologia e Insumos Estratégicos. Departamento de Assistência Farmacêutica e Insumos Estratégicos. Política Nacional de Plantas Medicinais e Fitoterápicos. Brasília: Ministério da Saúde, Brasília. 1a edição. 2006; 136 p.

7. BRASIL. Ministério da Saúde. Secretaria de Atenção à Saúde. Departamento de Atenção Básica. Política Nacional de Práticas Integrativas e Complementares no SUS: atitude de ampliação de acesso. Brasília: Ministério da Saúde, 2015; 96p.

8. BRASIL. Ministério da Saúde. RDC n 48, de 16 de março de 2004. Dispõe sobre o registro de medicamentos fitoterápicos. Disponível em: http://portal.anvisa.gov.br. Acesso em: 17 jan. 2019.

9. BRASIL. Ministério da Saúde. Notícias SESAI: Indígenas são atendidos no polo base de Oriximiná terão nova casa de saúde. Disponível em: http://portalms.saude.gov.br/noticias/sesai/44679-indigenas-atendidos-no-polo-base-de-oriximina-terao-novacasa-de-saude

10. CASTRO NJC, et al. Inclusão de disciplinas em graduação de enfermagem sobre populações tradicionais amazônicas. Revista Cogitare Enfermagem. 2017; 22(2): e49730.

11. GONTIJO MBA, NUNES MF. Práticas integrativas e complementares: conhecimento e credibilidade de profissionais do serviço público de saúde. Trab. educ. Saúde, 2017; 15(1): 301-320.

12. IBGE. Instituto Brasileiro de Geografia e Estatística. Oriximiná. Disponível em: https://cidades.ibge.gov.br/brasil/pa/oriximina/panorama

13. LEDA PHO, et al. Plantas medicinais e agentes comunitários de saúde de oriximiná: uma analise preliminar do conhecimento acerca do tema. Universidade do Oeste do Pará. VI Jornada Acadêmica. 2017

14. LÉDA PHO, et al. Plantas medicinais e fitoterapia em Oriximiná-Pará, Brasil: percepção e intenção de uso pelos profissionais do Sistema Único de Saúde (SUS). VITTALLE-Revista de Ciências da Saúde, 2018; 30(1): 11-25.

15. MOTTA FILHO SC. Direito Constitucional: teoria, jurisprudência. 12ํed. Rio de Janeiro: Impetus, 2003. P. 143

16. OLIVEIRA DR, et al. Autorização de Acesso ao Conhecimento Tradicional Associado com fins de Bioprospecção: O Caso da UFRJ e da Associação de Comunidades Quilombolas de Oriximiná-ARQMO. Revista Fitos Eletrônica, 2013; 5(1): 59-76.

17. OMS. Organização Mundial de Saúde. Fundo das Nações Unidas para Infância (UNICEF). Conferência internacional sobre cuidados primários de saúde, Alma-Ata-URSS, 6-12 de setembro de 1978.

18. PEREIRA MGS, COELHO-FERREIRA M. Uso e diversidade de plantas medicinais em uma comunidade quilombola na Amazônia Oriental, Abaetetuba, Pará. Biota Amazônia (Biote Amazonie, Biota Amazonia, Amazonian Biota), 2017; 7(3): 5768.

19. PLÁCIDO AL, et al. Percepção dos Gestores das Unidades Básicas de Saúde Sobre as Práticas Integrativas e Complementares. Revista Multidisciplinar e de Psicologia, 2019; 13(43): 865-872.

20. SILVA ASP, FEITOSA ST. Revisão sistemática evidencia baixo nível de conhecimento acerca da política nacional de práticas integrativas e complementares por parte de gestores e profissionais da saúde. VITTALLE-Revista de Ciências da Saúde, 2018; 30(1): 105-114. 